\title{
ON THE ITERATED MEAN TRANSFORMS OF OPERATORS
}

\author{
Sungeun Jung, Eungil Ko And MeE-Jung LeE
}

Abstract. Let $T=U|T|$ be the polar decomposition of an operator $T \in \mathscr{L}(\mathscr{H})$. For given $s, t \geqslant 0$, we say that $\widehat{T}_{s, t}:=s U|T|+t|T| U$ is the weighted mean transform of $T$. In this paper, we study properties of the $k$-th iterated weighted mean transform $\widehat{T}_{s, t}^{(k)}$ of $T=U|T|$ when $U$ is unitary. In particular, we give the polar decomposition of such $\widehat{T}_{s, t}^{(k)}$ and investigate its applications. Finally, we consider the iterated weighted mean transforms of a weighted shift.

Mathematics subject classification (2010): 47B49, 47B20, 47B37. subspaces.

Keywords and phrases: Weighted mean transform, Duggal transform, polar decomposition, invariant

\section{REFERENCES}

[1] A. Aluthge, On p-hyponormal operators for $0<p<1$, Inter. Equ. Oper. Theory 13(1990), 307315.

[2] S. K. Berberian, Approximate proper vectors, Proc. Amer. Math. Soc. 13(1962), 111-114.

[3] I. COlojoARĂ AND C. FoiAş, Theory of generalized spectral operators, Gordon and Breach, New York, 1968.

[4] C. Foiaş, I. B. Jung, E. Ko And C. Pearcy, Complete contractivity of maps associated with the Aluthge and Duggal transforms, Pacific J. Math. 209(2003), 249-259.

[5] M. ITO, T. YAMAZAKI AND M. YANAGIDA, On the polar decomposition of the Aluthge trasformation and related results, J. Operator Theory 51(2004), 303-319.

[6] I. B. Jung, E. Ko AND C. PeARCY, Aluthge transforms of operators, Inter. Equ. Oper. Theory 37(2000), 449-456.

[7] I. B. JUnG, E. Ko AND C. PEARCY, Spectral pictures of Aluthge transforms of operators, Inter. Equ. Oper. Theory 40(2001), 52-60.

[8] I. B. Jung, E. Ko AND C. PEARCY, The iterated Aluthge transform of an operator, Inter. Equ. Oper. Theory 45(2003), 375-387.

[9] S. JUng, E. Ko AND S. PARK, Subscalarity of operator transforms, Math. Nachr. 288(2015), 20422056.

[10] E. Ko And M. LEE, On backward Aluthge iterates of hyponormal operators, Math. Inequal. Appl. 18(2015), 1121-1133.

[11] S. Lee, W. Lee And J. Yoon, The mean transform of bounded linear operators, J. Math. Anal. Appl. 410(2014), 70-81.

[12] K. B. Laursen And M. M. Neumann, Introduction to Local spectral theory, London Math. Soc. Monograghs New Series. Claredon Press, Oxford, 2000.

[13] S. Mathew and M. S. Balasubramani, On the polar decomposition of the Duggal transformation and related results, Oper. Matrices 3(2009), 215-225.

[14] M. PutinaR, Hyponormal operators are subscalar, J. Operator Theory 12(1984), 385-395.

[15] H. RadjaVi AND P. Rosenthal, Invariant subspaces, Springer-Verlag, 1973.

[16] D. XIA, Spectral theory of hyponormal operators, Springer Basel AG, Birkhäuser, 1983. 